\title{
Regeneration of toxigenic Pasteurella multocida induced severe turbinate atrophy in pigs detected by computed tomography
}

Tibor Magyar $^{1 *}$, Tamás Donkó², Imre Repa² and Melinda Kovács ${ }^{3}$

\begin{abstract}
Background: Atrophic rhinitis is a widely prevalent infectious disease of swine caused by Bordetella bronchiseptica and Pasteurella multocida. The course of the disease is considered to be different depending on the principal aetiological agents distinguishing B. bronchiseptica induced non-progressive and toxigenic $P$. multocida produced progressive forms. In order to compare the pathological events of the two forms of the disease, the development of nasal lesions has longitudinally been studied in pigs infected by either B. bronchiseptica alone or B. bronchiseptica and toxigenic $P$. multocida together using computed tomography to visualise the nasal structures.

Results: $B$. bronchiseptica infection alone caused moderately severe nasal turbinate atrophy and these lesions completely regenerated by the time of slaughter. Unexpectedly, complete regeneration of the bony structures of the nasal cavity was also observed in pigs infected by $B$. bronchiseptica and toxigenic $P$. multocida together in spite of seeing severe turbinate atrophy in most of the infected animals around the age of six weeks.

Conclusions: B. bronchiseptica mono-infection has been confirmed to cause only mild to moderate and transient lesions, at least in high health status pigs. Even severe turbinate atrophy induced by B. bronchiseptica and toxigenic P. multocida combined infection is able to be reorganised to their normal anatomical structure. Computed tomography has further been verified to be a useful tool to examine the pathological events of atrophic rhinitis in a longitudinal manner.
\end{abstract}

Keywords: Atrophic rhinitis, Bordetella bronchiseptica, Pasteurella multocida, Computed tomography

\section{Background}

Atrophic rhinitis (AR) is a widespread infectious disease of swine characterised by twisting or shortening of the snout [1]. This facial deformity results from an underlying atrophy of the nasal turbinate bones, which is the principal pathological lesion of AR. Two infectious agents are associated with the aetiology of AR: toxigenic strains of Bordetella bronchiseptica and Pasteurella multocida. A heat-labile toxin of P. multocida (PMT) plays a dominant role in developing the characteristic lesions of AR, and so toxigenic $P$. multocida is considered to be the primary aetiological agent of the disease. On the other hand, a substantial body of evidence shows that $P$. multocida is unable to fulfil its role without predisposing circumstances

\footnotetext{
*Correspondence: magyar.tibor@agrar.mta.hu

'Institute for Veterinary Medical Research, Centre for Agricultural Research,

Hungarian Academy of Sciences, Budapest, Hungary

Full list of author information is available at the end of the article
}

of which B. bronchiseptica pre-infection is the most commonly recognised $[2,3]$. Because of this specific synergistic interaction between $B$. bronchiseptica and P. multocida AR is classified as a genuine polymicrobial disease [4].

Although both B. bronchiseptica and P. multocida have the ability to cause turbinate atrophy in pigs, a number of research findings show that the severity and persistence of the pathological changes generated by these two bacteria are different. B. bronchiseptica infection has been reported to result in mild-to-moderate lesions that could regenerate by the time pigs reach slaughter weight $[5,6]$, while toxigenic strains of $P$. multocida proved to be able to produce severe and irreversible turbinate atrophy $[2,7]$. These observations led to the proposition to distinguish non-progressive and progressive forms of AR according to the principal aetiological agents that can be found in the herd in question [8]. Nevertheless, no exact description of the course of the disease from infection in 
a young age until reaching slaughter weight is available in the literature.

In early longitudinal studies of AR, large numbers of pigs were used because several animals had to be euthanized at selected time-points in order to obtain a satisfactory number of cross-sectional snout slices to perform the evaluations. Moreover, this approach provided no data about the changes in individual animals over time. These problems were overtaken by the introduction of computed tomographic imaging that allowed non-invasive tracking of the pathogenesis of AR within single pigs examining them at several time-points without the need to sacrifice their life [9-11].

The purpose of this study was to follow the morphological changes of nasal structures in pigs infected by either B. bronchiseptica alone or B. bronchiseptica and toxigenic $P$. multocida together using computed tomography (CT) for visualising the nasal cavities of the experimental animals.

\section{Methods}

\section{Experimental animals}

The experiments complied with current Hungarian legislation and were authorised by the Food Chain Safety and Animal Health Directorate of the Somogy County Agricultural Office (Ref: 406-6/2002) based on the approval of the Ethics Committee of the Ministry of Agriculture.

Twenty-eight new-born piglets from a single closed herd were selected for each experiment. Clinical signs of AR had never been observed in the herd and toxigenic $P$. multocida had never been identified. No vaccination against AR had ever been undertaken on the farm. The P. multocida-free status of the selected animals was confirmed prior to initiating the study. Medicated early weaning was applied to obtain B. bronchiseptica-free piglets. The sows delivering the piglets had been treated with tulathromycin (Draxxin inj., Pfizer, New York, USA) administered in a dose of $1 \mathrm{ml} / 40 \mathrm{~kg}$ one week before farrowing. The piglets were moved to isolated units at 1-2 days of age where they were kept in heated compartments. Pigs were fed with Sprayfo milk replacer (Sloten, Deventer, Netherlands) until the age of 28 days using Mambo milk applicator device (Sloten, Deventer, Netherlands) according to the manufacturer's recommendations. At the age of 28 days the piglets were weaned from milk replacer and then consumed only prestarter feed. From 6 to 10 weeks of age they were fed with starter diet, and then fattening diet until the termination of the study. Animals were fed ad libitum from self-feeders and had free access to drinking water. No treatments were permitted that could have interfered with the outcome of the trial, such as the use of immunosuppressive drugs or antibiotics affecting the respiratory tract.

\section{Bacterial strains}

Toxigenic strain KM22 of B. bronchiseptica was isolated from a herd with clinical AR. LFB3, a toxigenic isolate of P. multocida, was kindly supplied by Dr JM Rutter (Institute for Animal Health, Compton, UK). Both strains are known to produce the disease [12]. B. bronchiseptica strain KM22, cultured on Bordet-Gengou (BG) agar (Oxoid, Basingstoke, UK) at 37C for 24 hours, was diluted in phosphate-buffered saline (PBS), $\mathrm{pH} 7.2$, to give a suspension of about $10^{6}$ Colony Forming Units $/ \mathrm{ml}(\mathrm{CFU} / \mathrm{ml})$. P. multocida strain LFB3, cultured on 5\% sheep blood agar (BA) at $37 \mathrm{C}$ for 24 hours, was diluted in Brain Heart Infusion (BHI) broth (Difco, Detroit, USA) to give a suspension of about $10^{8} \mathrm{CFU} / \mathrm{ml}$. The toxin-producing ability of $P$. multocida strain LFB3 was confirmed by a membrane assay [13].

\section{Experimental design}

Two experiments were performed. Each experiment consisted of two groups of 14 randomly selected piglets. Group 1 piglets were infected with either B. bronchiseptica alone or B. bronchiseptica and P. multocida together. Group 2 piglets remained uninfected (negative) controls. The day of $B$. bronchiseptica infection was study day 0 (D0) of the study.

Piglets in Group 1 in Experiment 1 were infected with B. bronchiseptica at 4 days of age by instilling $0.5 \mathrm{ml}$ of bacterial suspension into each nostril. Piglets in Group 1 in Experiment 2 were infected with $B$. bronchiseptica at 4 days of age by instilling $0.5 \mathrm{ml}$ of bacterial suspension into each nostril, and with $P$. multocida at 8 days of age by the same method.

Experiment 1 was terminated at 132 days of age and Experiment 2 at 128 days of age. At termination, the pigs were slaughtered and examined in a blind manner for turbinate bone atrophy (TA), nasal septum deviation (NSD) and lung lesions.

\section{Collection and bacteriologic examination of nasal secretions}

Nasal swabs were taken from piglets at each CT examination. The samples were tested for the presence of $B$. bronchiseptica on MacConkey agar (BBL, Sparks, USA) as described elsewhere [14]. The presence of $P$. multocida toxin in the primary cultures of nasal swabs was determined using a PMT ELISA (Oxoid, Hampshire, UK) according to the manufacturer's recommendation.

\section{Computer tomography}

To immobilise the pigs for the scanning procedure, $4 \mathrm{mg} / \mathrm{kg}$ azaperone (Stresnil, Janssen Pharmaceutica) was administered intramuscularly for sedation and $9 \mathrm{mg} / \mathrm{kg}$ ketamine hydrochloride (SBH-Ketamin inj., SelBruHa) was administered intravenously to anaesthetise the pigs. 
The anaesthetised pigs were fixed in stretched position, lying flat in a purpose-designed container during the CT examination. CT images from each pig were acquired using a Somatom Plus 40 (Siemens, Erlangen, Germany) third generation scanner. The starting position of the scanning was set approximately $10 \mathrm{~mm}$ anterior from the level of the first upper premolar teeth, and then consecutive three $\mathrm{mm}$ scans were taken with a table feed of five $\mathrm{mm}$. The imaging protocol was a so-called "high" algorithm, which is extremely sensitive in visualising tissues of high-density differences. Zoom factor was set to 3.5.

In Experiment 1, CT images of all pigs were acquired at study days 0, 9, 41, 70, 101, and 132 while in Experiment 2 they were obtained at study days $0,4,18,25,32,60,88$, and 128. Non-infected pigs were always imaged prior to the infected pigs to avoid cross-contamination.

\section{Interpretation of $\mathrm{CT}$ images}

Visual scoring of the nasal turbinate bones was done on CT scans at the level of the first premolar teeth. Each of the four scrolls of the ventral turbinate bones was scored according to the following criteria (TA score): 0 , no lesion; 1 , a small part of the turbinate bone (nearly half a scroll) is absent; 2, slight atrophy - more than half a scroll is absent; 3, moderate atrophy - the turbinate bone is straightened; 4, severe atrophy - total disappearance of the turbinate bone. NSD was scored on a scale of $0-2$ : 0 , normal; 1 , slight deviation; 2 , severe deviation. TA and NSD scores were summed for each individual to a maximum value of 18 (nasal lesion score, NLS).

\section{Results}

\section{Animal health and bacteriology}

B. bronchiseptica infected piglets did not show noteworthy clinical signs: only sporadic sneezing, mild nasal discharge and lacrimation were observed in some of the animals. In Experiment 2, B. bronchiseptica infection produced the same results while pronounced sneezing was noticed in all infected piglets from 2 days after the P. multocida infection. It lasted approximately one week then gradually disappeared.

Three pigs were euthanized for welfare reasons because of emaciation and weakness during their first weeks of life. No connection was established between these signs and the experimental infection.

B. bronchiseptica infected animals (Experiment 1) produced $B$. bronchiseptica culture positive samples up to D41 post infection. Most of the B. bronchiseptica P. multocida infected animals (Experiment 2) yielded $B$. bronchiseptica positive samples until D60. In the control pigs neither culture positive $B$. bronchiseptica isolation nor PMT identification was produced from their samples. The B. bronchiseptica infected pigs (Experiment 1) were also PMT negative throughout the whole observation period. PMT monitoring of the P. multocida infected pigs during each imaging did not result in consistent detection of PMT (Table 1).

\section{Assessment of CT images}

No pathological alterations of the nasal structures were noticed in the uninfected control pigs.

In both infected groups (Experiment 1 and 2), regressive changes were observed in the nasal architecture (Tables 2 and 3). However, the progression of these changes showed great individual variability. B. bronchiseptica infected pigs produced lower scores and greater diversity of the lesions than the pigs in the B. bronchiseptica - P. multocida infected group in which more even lesions and higher scores were recorded (Figure 1).

Only 4 out of 11 live piglets had 8 or higher NLS in the $B$. bronchiseptica infected group on D41 of the study. The lesions started to quickly regenerate after D41 and all animals became practically free of lesions until D101, i.e. the end, of the study. Figure 2 shows a pig having the highest NLS in this group at D41 and producing complete regeneration of the lesion by the time of termination.

In the B. bronchiseptica - P. multocida infected group, 12 out of the 14 piglets had 12 or higher NLS by the time of D25 indicating rapidly developing and severe turbinate atrophy in the whole group. Interestingly, most of the pigs showed unexpected signs of reparation noticed from D60 and at the time of the termination of the study

Table 1 Detection of P. multocida toxin (PMT) in piglets in group 1 in experiment 2

\begin{tabular}{ccccccccc}
\hline Pig ID & \multicolumn{7}{c}{ Post infection day } \\
\cline { 2 - 8 } & $\mathbf{0}$ & $\mathbf{4}$ & $\mathbf{1 8}$ & $\mathbf{2 5}$ & $\mathbf{3 2}$ & $\mathbf{6 0}$ & $\mathbf{8 8}$ & $\mathbf{1 2 8}$ \\
\hline 4 & - & - & + & - & + & - & - & - \\
5 & - & - & + & + & - & - & - & - \\
9 & - & - & + & + & - & - & - & - \\
12 & - & - & - & + & + & - & - & - \\
13 & - & - & - & + & + & - & - & - \\
16 & - & - & - & + & - & + & - & - \\
19 & - & - & + & - & + & - & - & - \\
21 & - & - & + & - & + & + & - & - \\
22 & - & - & + & + & + & - & - & - \\
25 & - & - & - & + & - & + & - & - \\
27 & - & - & - & + & + & - & - & - \\
29 & - & - & + & - & + & - & - & - \\
30 & - & - & - & + & + & - & - & - \\
32 & - & - & + & - & - & - & - & - \\
\hline
\end{tabular}

The piglets were infected intranasally with $B$. bronchiseptica at the age of 4 days (D0) and $P$. multocida at the age of 8 days (D4). PMT was shown out with a PMT ELISA (Oxoid, Hampshire, UK). 
Table 2 Nasal lesion scores of piglets in group 1 in experiment 1

\begin{tabular}{ccccccc}
\hline Pig ID & \multicolumn{5}{c}{ Post infection day } \\
\cline { 2 - 6 } & $\mathbf{0}$ & $\mathbf{9}$ & $\mathbf{4 1}$ & $\mathbf{7 0}$ & $\mathbf{1 0 1}$ & $\mathbf{1 3 2}$ \\
\hline 1 & 0 & 8 & Died & & & \\
2 & 0 & Died & & & & \\
3 & 0 & Died & & & & \\
4 & 0 & 3 & 3 & 2 & 1 & 0 \\
5 & 0 & 3 & 3 & 0 & 0 & 0 \\
6 & 0 & 1 & 1 & 0 & 0 & 0 \\
7 & 0 & 0 & 12 & 5 & 1 & 0 \\
9 & 0 & 4 & 11 & 3 & 1 & 0 \\
10 & 0 & 3 & 8 & 4 & 3 & 2 \\
13 & 0 & 0 & 2 & 1 & 0 & 0 \\
16 & 0 & 0 & 0 & 1 & 0 & 0 \\
21 & 0 & 6 & 8 & 2 & 1 & 1 \\
22 & 0 & 1 & 0 & 0 & 0 & 0 \\
24 & 0 & 4 & 6 & 0 & 0 & 0 \\
Means & 0 & 2,8 & 4,9 & 1,6 & 0,6 & 0,3 \\
\hline
\end{tabular}

The piglets were infected intranasally with $B$. bronchiseptica at the age of 4 days (D0).

(D128) 7 out of the 14 pigs had a score of $\leq 3$. Figure 3 demonstrates an example of such a complete regeneration of turbinate atrophy with a score of 15 on D25 and 32. Figure 4 illustrates the only case when the severe TA and NSD lasted until the termination of the study.

Table 3 Nasal lesion scores of piglets in group 1 in experiment 2

\begin{tabular}{ccccccccc}
\hline Pig ID & \multicolumn{7}{c}{ Post infection day } \\
\cline { 2 - 8 } & $\mathbf{0}$ & $\mathbf{4}$ & $\mathbf{1 8}$ & $\mathbf{2 5}$ & $\mathbf{3 2}$ & $\mathbf{6 0}$ & $\mathbf{8 8}$ & $\mathbf{1 2 8}$ \\
\hline 4 & 0 & 2 & 12 & 15 & 15 & 3 & 2 & 0 \\
5 & 0 & 3 & 12 & 12 & 10 & 0 & 0 & 1 \\
9 & 0 & 0 & 2 & 6 & 6 & 1 & 1 & 0 \\
12 & 0 & 0 & 11 & 14 & 13 & 12 & 12 & 7 \\
13 & 0 & 0 & 6 & 8 & 7 & 1 & 0 & 0 \\
16 & 0 & 1 & 13 & 13 & 14 & 11 & 8 & 9 \\
19 & 0 & 1 & 15 & 14 & 14 & 9 & 3 & 3 \\
21 & 0 & 2 & 13 & 15 & 15 & 15 & 11 & 11 \\
22 & 0 & 3 & 14 & 14 & 16 & 17 & 18 & 16 \\
25 & 0 & 3 & 14 & 14 & 16 & 8 & 5 & 9 \\
27 & 0 & 4 & 14 & 14 & 14 & 5 & 3 & 4 \\
29 & 0 & 5 & 9 & 12 & 12 & 4 & 5 & 3 \\
30 & 0 & 4 & 14 & 14 & 14 & 15 & 11 & 6 \\
32 & 0 & 0 & 9 & 12 & 10 & 1 & 0 & 0 \\
Means & 0,0 & 2,0 & 11,3 & 12,6 & 12,6 & 7,3 & 5,6 & 4,9 \\
\hline
\end{tabular}

The piglets were infected intranasally with $B$. bronchiseptica at the age of 4 days (D0) and P. multocida at the age of 8 days (D4).

\section{Discussion}

Our results confirmed the suggestion by a number of authors $[2,5,6,15]$ that $B$. bronchiseptica infection alone causes only moderately severe atrophy of the nasal turbinate bones and these lesions can completely regenerate over time. The pathological changes produced in the present study progressed until about 6 weeks of age, and then remarkable recovery was seen 30 days later followed by complete healing by the time of slaughter.

Former B. bronchiseptica challenge experiments showed conflicting results as to the degree of turbinate atrophy found in the infected pigs. One possible explanation could have been that variations in virulence of the various B. bronchiseptica strains may influence the outcome of the infection. However, Rutter et al. [16] could not demonstrate such differences among $B$. bronchiseptica strains independently whether they had been isolated from herds with or without AR. It seems more likely that the severity of the lesions is influenced by the health status of the piglets used in the study. In caesarean-derived, colostrum-deprived pigs [17] or gnotobiotic piglets [14], B. bronchiseptica infection resulted in moderate turbinate atrophy while severe lesions were developed in AR free but otherwise conventional piglets using the same B. bronchiseptica strain (B58) [18]. Conventional piglets might be more susceptible to $B$. bronchiseptica or other factors of the porcine respiratory disease complex may be involved in the disease process and promote the pathological effect exerted by $B$. bronchiseptica infection. We also used high health status animals in this study obtained by a method that followed the principles described by Alexander et al. [19] that may explain the low nasal lesion scores detected in the infected piglets.

It is generally accepted that toxigenic $P$. multocida causes irreversible nasal choncal hypoplasia. Therefore, it was a rather unexpected finding that even complete regeneration of the bony structures of the nasal cavity could be observed in pigs which had received a $B$. bronchiseptica and toxigenic P. multocida combined challenge in spite of the fact that severe turbinate atrophy was seen in most of the infected animals at 25 and 32 days after the first infection. Although bacteriological examination has not clearly verified the persistent colonisation of the nasal mucosa by toxigenic $P$. multocida, the success of the combined infection has been indicated by the development of these characteristic lesions. Thirty days later, however, significant reparation of the lesions was seen in 9 out of the 14 infected pigs. At the same time, in one pig the characteristic degree of progressive turbinate atrophy and severe nasal septum deviation persisted until termination of the observation period. No differences in any examined parameter, including bacteriological findings, could be found in this pig to explain why the lesions progressed until the end of the experiment. 


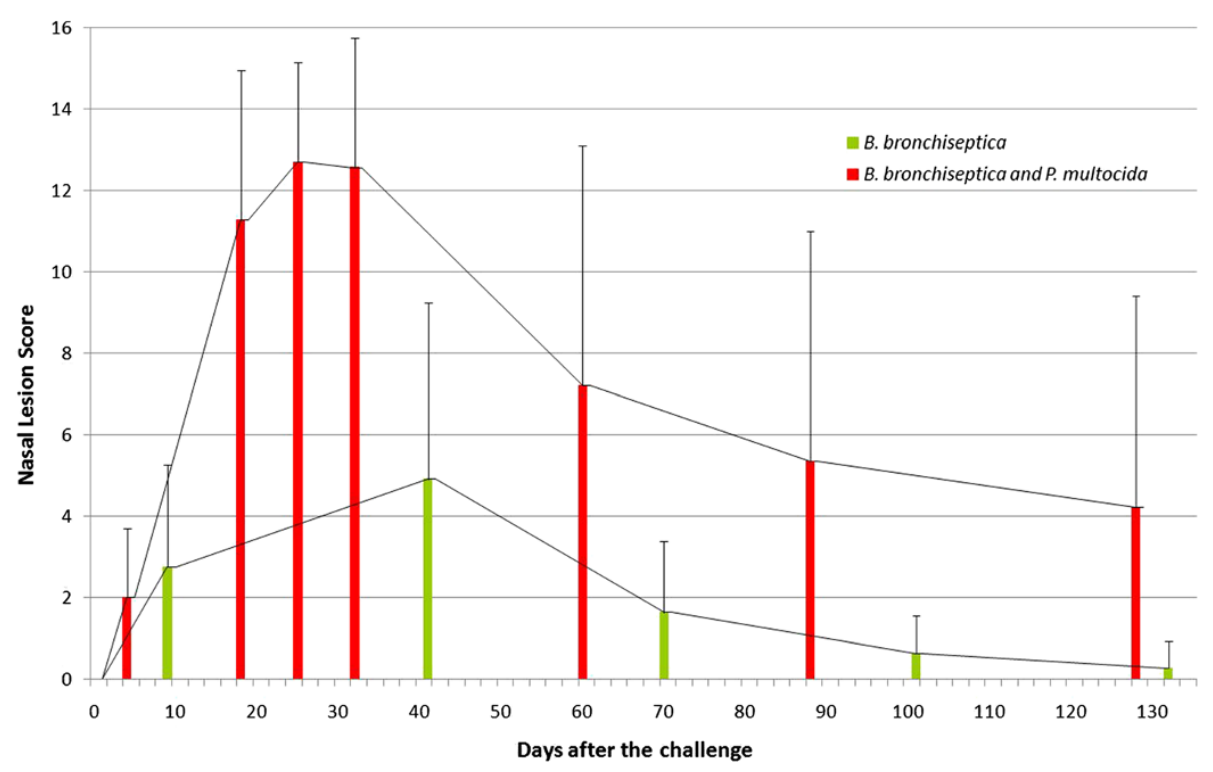

Figure 1 Development of nasal lesion scores in the infected piglets. The piglets were infected with either $B$. bronchiseptica alone (Group 1, Experiment 1) or B. bronchiseptica and P. multocida together (Group 1, Experiment 2). The diagram shows the mean nasal lesions scores calculated from the individual scores of the computed tomography scans gained throughout the observation period.

It is also remarkable that, on average, the highest degree of turbinate atrophy has been detected around the age of six weeks in both infected groups which is the conventional time for nasal lesion scoring in vaccine efficacy studies performed according to the requirements of the European Pharmacopoeia. It means that the timing of the evaluation is the most probable stage at which the lesions reach their maximum degree, and thus it appears to be optimal for measuring the effectiveness of AR vaccines. On the other hand, it seems questionable how the disease would proceed after this age that may be worth further studies to clarify its impact on the assessment of the protective value of the vaccines.

Previously, Jolie et al. [9] who first applied CT for the diagnosis of porcine AR reported a gradual decline in nasal lesion score occurred in one pig selected from a

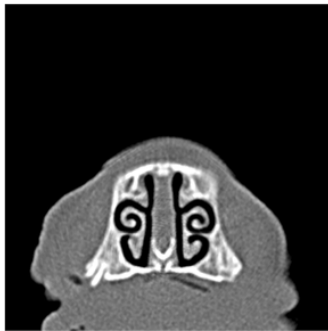

d0

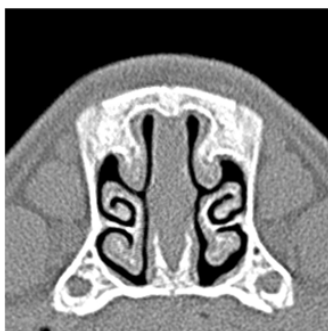

d70

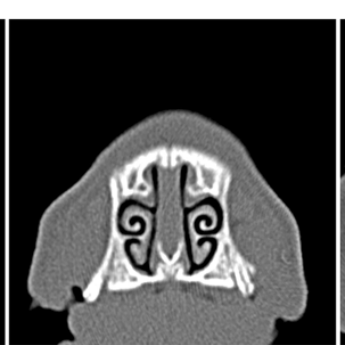

d9

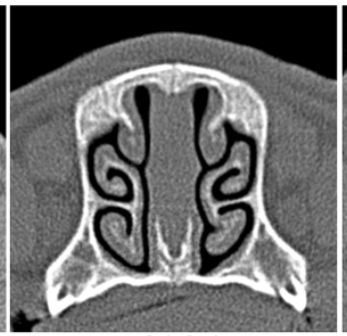

d101

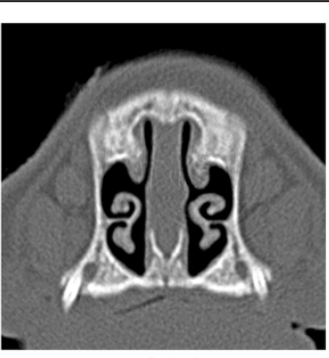

d41

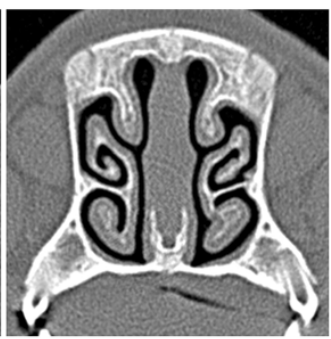

d132

Figure 2 Sequential computed tomography scans of pig No. 7 in Experiment 1. Pig No. 7 was infected with B. bronchiseptica at the age of 4 days (D0). This pig showed the most severe nasal turbinate atrophy (nasal lesion score 12) in this group that completely regenerated until Day 101 of the study. 


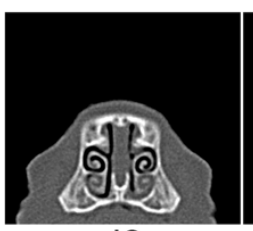

d0

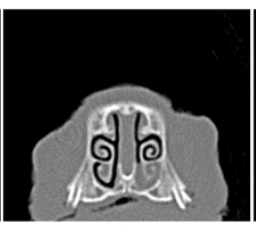

d4

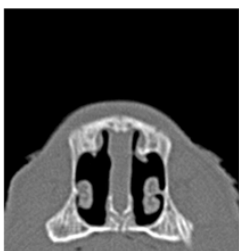

d32

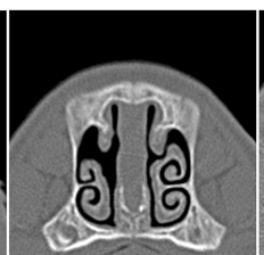

d60

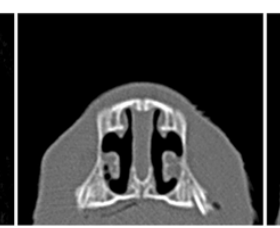

d18

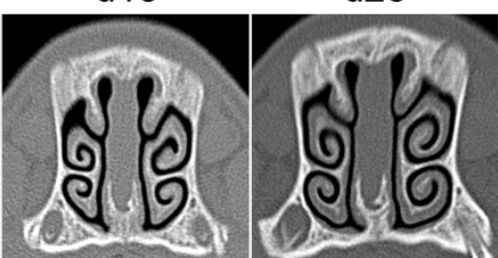

d88

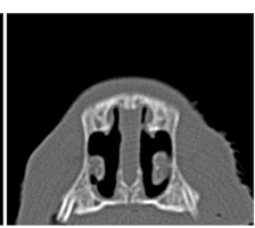

d25

d128

Figure 3 Sequential computed tomography scans of pig No. 4 in Experiment 2. Pig No. 4 was infected with B. bronchiseptica at the age of 4 days (D0) and P. multocida at the age of 8 days (D4). Complete regeneration of the nasal turbinates was observed although severe turbinate atrophy (nasal lesion score 15) was seen from Day 18 to Day 32.

commercial herd with clinically apparent atrophic rhinitis examined from three weeks of age until slaughter. However, neither P. multocida nor B. bronchiseptica was isolated from nasal swab samples and thus the exact aetiology and the form of AR represented by this case could not be established. Therefore, this is the first adequately supported demonstration that nasal turbinate bones may regenerate even from a stage of full score atrophy characteristic for the progressive form of AR. It also means that the definition of progressive AR may need some reconsideration. Our results strongly suggest that a heavy infection with PMT producing P. multocida assisted by previous $B$. bronchiseptica infection is not necessarily sufficient for the induction of severe and irreversible turbinate atrophy seen in conventional pigs examined at slaughter from AR positive herds. AR as a part of the porcine respiratory disease complex has been categorised as a multifactorial disease in which the two well-identified pathogens (B. bronchiseptica and toxigenic $P$. multocida) are working together with a number of predisposing factors like additional infectious agents, housing problems or poor management. Further studies are needed to recognise other factor or factors of possible importance and to understand their role in the aetiology and pathogenesis of AR.

CT has proved to be a feasible tool for such investigations. The unceasing development of $\mathrm{CT}$ equipments have resulted in continuously improving image resolution that, together with increasing scanning speed, makes possible the examination of smaller and smaller details. Its ability to follow the course of the disease in

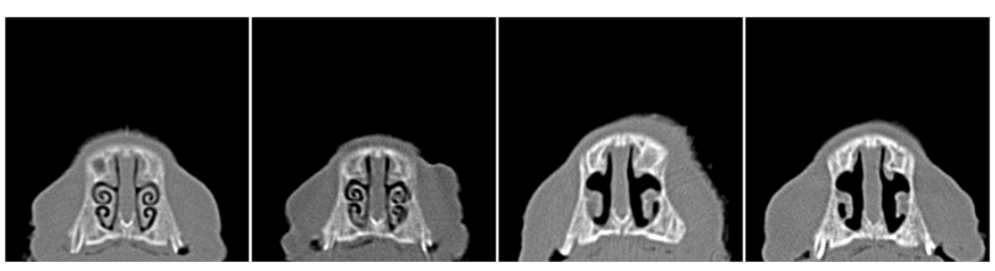

d0 d4 d18 d25

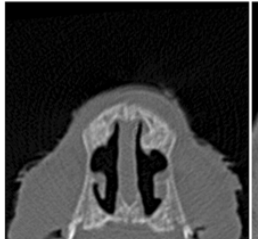

d32

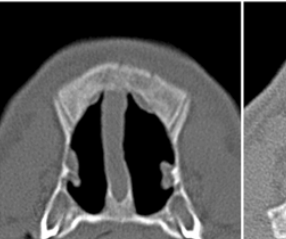

d60

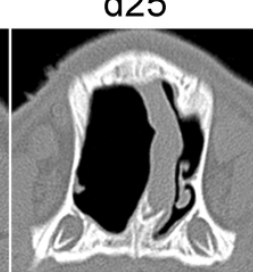

d88

Figure 4 Sequential computed tomography scans of pig No. 22 in Experiment 2. Pig No. 22 was infected with B. bronchiseptica at the age of 4 days (D0) and P. multocida at the age of 8 days (D4). Severe turbinate atrophy and nasal septum deviation (nasal lesion score 16-18) developed that progressed throughout the whole observation period. 
the same animal without the need to sacrifice is of great importance as well as its potential application for studying other diseases in a longitudinal manner just as proved recently by early detection of pneumonia and monitoring the pathological events during its progression $[20,21]$.

\section{Conclusions}

The transient nature of $B$. bronchiseptica induced nasal turbinate lesions was confirmed in a longitudinal manner. B. bronchiseptica infection alone caused only mild to moderate lesions that completely regenerated over time, at least in the high health status pigs used in the study. Although B. bronchiseptica and toxigenic P. multocida combined infection induced severe turbinate atrophy, even these lesions were able to be reorganised to their normal anatomical structure suggesting that the pathogenesis of the progressive irreversible form of AR maybe more complicated than it has been assumed so far. CT is a very valuable device for a non-invasive tracking of the pathological events of AR examining the same animal at several occasions.

\section{Competing interests}

The authors declare that they have no competing interests.

\section{Authors' contributions}

TM conceived the study design, performed bacteriological procedures, participated in experimental work, data analysis and drafted the manuscript. TD had important input into experimental work, data collection and data analysis. IR coordinated the computed tomography examinations, contributed to data collection and data analysis. MK participated in working out the study design, coordinated the work and commented on the manuscript. All authors read and approved the final version of the manuscript.

\section{Acknowledgements}

The authors thank Dr. A. J. Lax for the useful comments on the manuscript. This work was supported by grant OTKA T034650.

\section{Author details}

'Institute for Veterinary Medical Research, Centre for Agricultural Research, Hungarian Academy of Sciences, Budapest, Hungary. ${ }^{2}$ Institute of Diagnostic Imaging and Radiation Oncology, Kaposvár University, Kaposvár, Hungary. ${ }^{3}$ Department of Physiology and Animal Hygiene, Faculty of Animal Science, Kaposvár University, Kaposvár, Hungary.

Received: 5 May 2013 Accepted: 28 October 2013

Published: 30 October 2013

\section{References}

1. Rutter JM: Atrophic rhinitis in swine. Adv Vet Sci Comp Med 1985, 29:239-279.

2. Pedersen $\mathrm{KB}$, Barfod $\mathrm{K}$ : The aetiological significance of Bordetella bronchiseptica and Pasteurella multocida in atrophic rhinitis of swine. Nord Vet Med 1981, 33:513-522.

3. Chanter N, Magyar T, Rutter JM: Interactions between Bordetella bronchiseptica and toxigenic Pasteurella multocida in atrophic rhinitis of pigs. Res Vet Sci 1989, 47:48-53.

4. Magyar T, Lax AJ: Atrophic rhinitis. In Polymicrobial Diseases. Edited by Brogden KA, Guthmiller JM. Washington (DC, USA): ASM Press; 2002:169-197.

5. Rutter JM: Quantitative observations on Bordetella bronchiseptica infection in atrophic rhinitis of pigs. Vet Rec 1981, 108:451-454.

6. Tornoe N, Nielsen NC: Inoculation experiments with Bordetella bronchiseptica strains in SPF pigs. Nord Vet Med 1976, 28:233-242.

7. Rutter JM, Rojas X: Atrophic rhinitis in gnotobiotic piglets: differences in the pathogenicity of Pasteurella multocida in combined infections with Bordetella bronchiseptica. Vet Rec 1982, 110:531-535.
8. De Jong MF: Progressive and nonprogressive atrophic rhinitis. In Diseases of Swine. 9th edition. Edited by Straw BE, Zimmerman JJ, D'Allaire S, Taylor DJ. Ames, lowa: Blackwell Publishing; 2006:577-602.

9. Jolie R, De Roose P, Tuyttens N: Diagnosis of atrophic rhinitis by computerised tomography: a preliminary report. Vet Rec 1990, 126:591-594.

10. Shryock T, Losonsky J, Smith W, Gatlin C, Francisco C, Kuriashkin I, Clarkson $\mathrm{R}$, Jordan W: Computed axial tomography of the porcine nasal cavity and a morphometric comparison of the nasal turbinates with other visualization techniques. Can J Vet Res 1998, 62:287-292.

11. Magyar T, Kovács F, Donkó T, Bíró H, Romvári R, Kovács M, Repa I: Turbinate atrophy evaluation in pigs by computed tomography. Acta Vet Hung 2003, 51:485-491.

12. Magyar T, Donkó $T$, Kovács F: Atrophic rhinitis vaccine composition triggers different serologic profiles that do not correlate with protection. Acta Vet Hung 2008, 56:27-40.

13. Magyar T, Rimler RB: Detection and enumeration of toxin-producing Pasteurella multocida with a colony-blot assay. J Clin Microbiol 1991, 29:1328-1332

14. Magyar T, Chanter N, Lax AJ, Rutter JM, Hall GA: The pathogenesis of turbinate atrophy in pigs caused by Bordetella bronchiseptica. Vet Microbiol 1988, 18:135-146.

15. Duncan JR, Ross RK, Switzer WP, Ramsey RK: Pathology of experimental Bordetella bronchiseptica infection in swine: atrophic rhinitis. Am J Vet Res 1966, 27:457-466.

16. Rutter JM, Francis LM, Sansom BF: Virulence of Bordetella bronchiseptica from pigs with or without atrophic rhinitis. J Med Microbio/ 1982, 15:105-116.

17. Brockmeier SL, Register KB, Magyar T, Lax AJ, Pullinger GD, Kunkle RA: Role of the dermonecrotic toxin of Bordetella bronchiseptica in the pathogenesis of respiratory disease in swine. Infect Immun 2002, 70:481-490.

18. Magyar $T$, King VL, Kovács F: Evaluation of vaccines for atrophic rhinitis a comparison of three challenge models. Vaccine 2002, 20:1797-1802.

19. Alexander TJL, Thornton K, Boon G, Lysons RJ, Gush AF: Medicated early weaning to obtain pigs free from pathogens endemic in the herd of origin. Vet Rec 1980, 106:114-119.

20. Pósa R, Donkó T, Bogner P, Kovács M, Repa I, Magyar T: Interaction of Bordetella bronchiseptica, Pasteurella multocida, and fumonisin B1 in the porcine respiratory tract as studied by computed tomography. Can J Vet Res 2011, 75:176-182.

21. Pósa R, Magyar T, Stoev SD, Glávits R, Donkó T, Repa I, Kovács A: Use of computed tomography and histopathologic review for lung lesions produced by the interaction between Mycoplasma hyopneumoniae and fumonisin mycotoxins in pigs. Vet Pathol 2013, DOl:10.1177/0300985813480510.

doi:10.1186/1746-6148-9-222

Cite this article as: Magyar et al:: Regeneration of toxigenic Pasteurella multocida induced severe turbinate atrophy in pigs detected by computed tomography. BMC Veterinary Research 2013 9:222.

\section{Submit your next manuscript to BioMed Central and take full advantage of:}

- Convenient online submission

- Thorough peer review

- No space constraints or color figure charges

- Immediate publication on acceptance

- Inclusion in PubMed, CAS, Scopus and Google Scholar

- Research which is freely available for redistribution 